\title{
Dr Charlton et al., Commentary and Complementary Data to Add to "Compliance with Cancer Quality Measures Over Time and Their Association with Survival Outcomes: The Commission on Cancer's Experience with the Quality Measure Requiring at Least 12 Regional Lymph Nodes to be Removed and Analyzed with Colon Cancer Resections"
}

\author{
Lawrence N. Shulman, MD \\ Abramson Cancer Center, University of Pennsylvania, Philadelphia, PA
}

Response to: Dr Charlton et al., Commentary and Complementary Data to Add to "Compliance with Cancer Quality Measures Over Time and Their Association with Survival Outcomes: The Commission on Cancer's Experience with the Quality Measure Requiring at Least 12 Regional Lymph Nodes to be Removed and Analyzed with Colon Cancer Resections"

We appreciate the commentary and complementary data provided by Dr Charlton and colleagues, who raise important issues to be considered. They document better compliance with the colon resection 12 node measure in programs that are Commission on Cancer (CoC) accredited, compared with those that are not. There are several possible explanations for this. It may be that programs that chose not to seek CoC accreditation, or sought it but were not accredited, are less focused on cancer care delivery overall and on the use of quality measures to assess their performance. It may also be that the $\mathrm{CoC}$ approach of automatically feeding back program performance on quality measures to their programs helps programs focus on their compliance with measures, and, when necessary, develop interventions to improve. This is an important principle of how $\mathrm{CoC}$ addresses quality at the program

(C) Society of Surgical Oncology 2019

First Received: 9 December 2019;

Published Online: 19 December 2019

L. N. Shulman, MD

e-mail: lawrence.shulman@uphs.upenn.edu level. As part of their accreditation process, CoC programs are required to report on their performance against a number of quality measures, including this one, on a regular basis, to their cancer committee. Annual performance against this and other measures is provided to programs through the $\mathrm{CoC}$ Cancer Quality Improvement Program report, which is specifically designated to be reviewed by the cancer committee and with hospital leadership. Programs that are not $\mathrm{CoC}$ accredited will not have easy access to data documenting their performance, and may not have it at all.

It is important to note, as the authors do, that there was improvement over time in the non-CoC-accredited programs, which of course is good. However, their performance remained significantly below that of $\mathrm{CoC}$ programs throughout the measurement period.

The authors note the challenges faced by rural programs seeking $\mathrm{CoC}$ accreditation, and in their management of cancer care and quality. We agree that $\mathrm{CoC}$ should specifically address smaller rural hospitals and investigate ways that $\mathrm{CoC}$ can help build strength and quality in their programs, while acknowledging that these programs face unique challenges that larger urban programs do not. Planning towards this end is underway.

Lawrence N. Shulman, MD, on behalf of the authors 